\section{Are leukotoxins toxic?}

To the editor - Moghaddam et al. reported in last month's issue that whereas leukotoxin exposure has no effect, metabolic conversion of leukotoxins to their corresponding diols by epoxide hydrolase is toxic to cells'. We suggest that leukotoxin may exert its cytotoxic effect through a mechanism other than transformation to diols.

Moghaddam and colleagues showed that leukotoxin diols are toxic to the cells of spodoptera frugiperdia transfected with epoxide hydrolase-bearing expression vectors and to cultured alveolar type II epithelial cells. They further demonstrate that conscious animals were more susceptible to toxicity induced by the diols as opposed to their precursors'. They conclude that soluble epoxide hydrolase can convert leukotoxins to their corresponding diols which are cytotoxic. This report is of importance in drawing attention to the biological effects of linoleate metabolites in contrast to the more intensively studied arachidonic acid metabolites. However, the observation that (iso)leukotoxins themselves may be protoxins seems to depend on the experimental system used. Leukotoxins affect many cell types. Our work suggests that leukotoxin may exert its cytotoxic effect through mechanisms other than transformation to diols by epoxide hydrolase ${ }^{2-s}$. The pulmonary endothelial cells used in our laboratory are an excellent model with which to study the acute respiratory distress syndrome, because biological effects are observed at 50 fold lower concentrations ${ }^{2}$ and much earlier times (within 20 minutes) than those reported by Moghaddam et al. 'Our preliminary results indicate that leukotoxins are toxic to cultured pulmonary endothelial cells via stimulation of concurrent production of NO and $\mathrm{O}$; , suggesting direct activation of vascular nitric oxide synthase. Our findings may identify another pathway of leukotoxin action because acute respiratory distress syndrome may be involved preferentially in the damage of pulmonary vascular endothelial cells. Although it is very difficult to exclude minor generation of the diols or other metabolites of leukotoxin in our experiments, Moghaddam et al.'s report and our findings reveal diverse and complicated mechanisms of lung injury induced by leukotoxin.

TAKESHI ISHIZAKI ${ }^{1} \&$ SHINGO AMFSHIMA ${ }^{1}$ 'Department of Internal Medicine

\section{Shigerd Matsukawa ${ }^{2}$ \\ 'Central Research Laboratory \\ Fukui Medical School, Shimoaizuki, \\ Matsuokacho, Fukui, Japan 910-11}

Hainmock replies - The work we reported is not in conflict with that of Ishizaki and colleagues and other groups working on the cardiovascular effects of leukotoxins. We do, however, caution the possibility that leukotoxin metabolites could be more potent than the parent epoxides in some systems. This caution is especially applicable to the epoxide functionality which can be readily converted to diols and glutathione conjugates. Indeed, we have not excluded metabolism of leukotoxin diols to still more active compounds. As with oxylipins in the arachidonate cascade, we might find activity associated with both the parent leukotoxin epoxides and their metabolites.

In our studies, the diols are more active than the epoxides. The pulmonary vascular endothelial cells used by Ishizaki $e t$ al. are likely involved in clinical symptoms of adult respiratory distress system and are used in a compelling bioassay. The leukotoxins are highly active in this system. However, it is much more difficult to exclude the diols as possible bioactive agents than it is to exclude the leukotoxins. Epoxides can be converted readily to diols, but reversing the reaction is difficult. Most cells contain some epoxide hydrolase. The diols are much more polar than the epoxides and may penetrate cells less rapidly. Finally, one can anticipate that the diols themselves will be readily degraded in many systems.

No potent, irreversible inhibitors of the soluble epoxide hydrolase exist. However, 4 and 4 '-substituted chalcone oxides are potent, transient inhibitors of the enzyme while the corresponding unsubstituted chalcone oxide is not. We can offer samples of chalcone oxides, leukotoxins and diols and plasmids for the human, rodent and plant soluble epoxide hydrolases to anyone interested in testing if the parent leukotoxins or their diol metabolites are active in their biological system.

Bruce. D. Hammock

Division of Entomology

C.SIRO Black Mountain Laboratory

GPO Box 1700 Canberra ACT 2601, Australia

1. Moghaddam, M.F. et al. Bioactivation of leukotoxins to their toxic diols by epoxide hydrolase. Nat. Med. 3, 562-566 (1997).

2. Ishizaki, T., Takahashi, H., Ozawa, T., Chang, S.-W \& Voelkel, N.F. Leukotoxin, 9, 10-epoxy-12-octadecenoate causes pulmonary vasodilation in rats. Am. I. Physiol. 268, 123-128 (1995).

3. Ishizaki, T. et al. Increased nitric oxide biosynthesis in leukotoxin, 9, 10-epoxy-12-octadecenoate injured lung. Biochem. Biophys. Res. Commun. 210, 133-137 (1995).

4. Ishizaki, $T$, et al. Leukotoxin, 9, 10-epoxy-12-octadecenoate causes edematous lung injury via activation of vascular nitric oxide synthase. Am. 1. Physiol. 269, 65-70 (1995).

5. Sakai, T. et al. Role of nitric oxide and superoxide anion in leukotoxin, 9, 10-epoxy-12-octadecenoate-induced mitochondrial dysfunction. Free Rad. Biol. \& Med. 20, 607-612 (1996).

\section{- WE'VE MOVED •}

\section{Our new address is:}

\section{Park Avenue South, 10th floor New York, NY 10010-1707}

Our new phone number is:

212.726.9325

Our new fax number is:

212.683.5751

Our new e-mail is: medicine natureny.com

\section{LETTERS TO THE EDITOR}

Nature Medicine encourages letters to the editor. Priority is given to short (less that 500 words) and provocative letters on issues of the broadest interest to the biomedical research community. Letters should be addressed to Natue Medicine, 345 Park Avenue South, New York, NY 100101707 , USA, or sent by fax $(212.683 .5751)$ or email (medicine@natureny.com). 